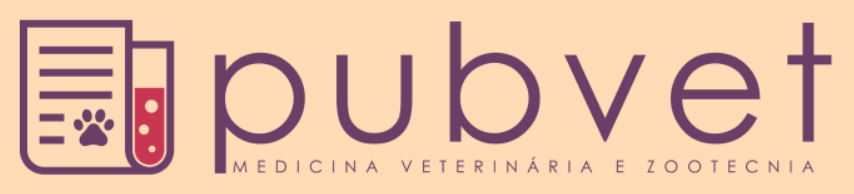

HTTP://DX.DOI.ORG/10.22256/PUBVET.V11N11.1132-1137

\title{
Parasitos gastrintestinais em gatos da cidade de Porto Alegre, Rio Grande do Sul
}

\author{
Sandra Márcia Tietz Marques ${ }^{1 *}$, Muriel Rodrigues Ferraz de Oliveira ${ }^{2}$, Mary Jane \\ Tweendie de Mattos Gomes
}

${ }^{1}$ Médica Veterinária, Departamento de Patologia Clínica Veterinária, Universidade Federal do Rio Grande do Sul. ${ }^{2}$ Graduanda em Medicina Veterinária, Universidade Federal do Rio Grande do Sul. E-mail: muriel_rfo@ @otmail.com ${ }^{3}$ Profa. Dra. Laboratório de Helmintoses, Universidade Federal do Rio Grande do Sul. E-mail: mary.gomes@ufrgs.br *Autor para correspondência: Faculdade de Veterinária, Universidade Federal do Rio Grande do Sul. Av. Bento Gonçalves, 9090. Bairro Agronomia, Porto Alegre, RS. CEP: 90540-000. Fax: +5551 3308.7305. E-mail: smtmuni@hotmail.com

RESUMO. O objetivo deste trabalho é relatar o parasitismo gastrointestinal em gatos atendidos no Hospital de Clínicas Veterinárias da Universidade Federal do Rio Grande do Sul (HCV/UFRGS), no período de 2014 a 2016. Foram analisadas amostras fecais de 339 felinos domiciliados da cidade de Porto Alegre, RS. As amostras foram processadas pelos métodos de Willis-Mollay, Lutz e Faust. Do total, 31,85\% (108) apresentaram resultado positivo. Os parasitos de maior prevalência foram Ancylostoma spp. (40,74\%), Cystoisospora spp. $(21,29 \%)$ e Toxocara spp. $(12,96 \%)$. Conclui-se que a prevalência mais alta ocorreu no ano de 2016, sem diferença para machos e fêmeas. Mesmo em baixa prevalência, parasitos dos gêneros Paragonimus, Spirometra e Giardia demandam atenção devido ao potencial zoonótico, o que torna o papel do médico veterinário de extrema importância participando da difusão e conscientização sobre a relevância destas parasitoses.

Palavras chave: felinos, helmintos, prevalência, parasitismo, zoonoses

\section{Gastrointestinal parasites in cats of the city of Porto Alegre, Rio Grande do Sul}

\begin{abstract}
The objective of this work is to determine the gastrointestinal parasitism in cats treated at the Hospital of Veterinary Clinics of the Universidade Federal do Rio Grande do Sul (HCV / UFRGS) from 2014 to 2016. Fecal samples from 339 felines domiciled in the city of Porto Alegre, RS, were analyzed. The samples were processed by the methods of Willis-Mollay, Lutz and Faust. Of the total, 31.85\% (108) presented a positive result. The most prevalent parasites were Ancylostoma spp. (40.74\%), Cystoisospora spp. $(21.29 \%)$ and Toxocara spp. (12.96\%). It is concluded that the highest prevalence occurred in the year 2016, with no difference for males and females. Even at low prevalence, parasites of the genus Paragonimus, Spirometra and Giardia demand attention due to the zoonotic potential, which makes the role of the veterinarian of extreme importance participating in the diffusion and awareness about the relevance of these parasitoses.
\end{abstract}

Keywords: feline, helminths, prevalence, parasitic diseases, zoonoses

\section{Parasitos gastrintestinales en gatos de la ciudad de Porto Alegre, Rio Grande do Sul}




\begin{abstract}
RESUMEN. El objetivo de este trabajo fue determinar el parasitismo gastrointestinal en gatos atendidos en el Hospital de Clínicas Veterinarias de la Universidade Federal do Rio Grande do Sul (HCV / UFRGS), en el período de 2014 a 2016. Se analizaron muestras fecales de 339 felinos domiciliados de la ciudad de Porto Alegre, RS. Las muestras fueron procesadas por los métodos de Willis-Mollay, Lutz y Faust. Del total, el 31,85\% (108) presentó un resultado positivo. Los parásitos de mayor prevalencia fueron Ancylostoma spp. (40,74\%), Cystoisospora spp. $(21,29 \%)$ y Toxocara spp. $(12,96 \%)$. Se concluye que la prevalencia más alta ocurrió en el año 2016, sin diferencia para machos y hembras. A pesar de la baja prevalencia, los parásitos de los géneros Paragonimus, Spirometra y Giardia demandan atención debido al potencial zoonótico, lo que hace el papel del médico veterinario de extrema importancia participando de la difusión y concientización sobre la relevancia de estos parásitos.
\end{abstract}

Palabras clave: felinos, helmintos, prevalencia, enfermedades parasitarias, zoonosis

\section{Introdução}

A verticalização das cidades afeta não somente a vida de seres humanos, como também dos animais domésticos que os acompanham. Com a crescente demanda por pets que estejam adaptados a ambientes menores e que possuam menos necessidades de cuidados por parte dos tutores, estes tem encontrado nos felinos um perfil de animal de estimação compatível com o progressivo processo de urbanização.

O estreitamento do convívio entre estes animais e o homem resulta em maior exposição a agente com potencial zoonótico. Os felinos são reservatórios de diversos parasitos, entre eles os helmintos gastrintestinais, que possuem fundamental importância em saúde pública por parasitarem não somente estes hospedeiros como também o homem (Mundim et al., 2004). Infecções como Larva Migrans Visceral e Larva Migrans Cutânea, causadas, respectivamente, pelos agentes Toxocara spp. e Ancylostoma braziliense são exemplos de zoonoses parasitárias transmitidas por gatos (Schantz, 1991, Gennari et al., 1999). As protozooses também estão inclusas devido a importância de gêneros Giardia e Cystoisospora, responsáveis por afecções devido a alterações produzidas na mucosa intestinal (Tesserolli et al., 2005, Quadros et al., 2015).

Por conta da proximidade do felino com o homem, torna-se fundamental o correto controle destas parasitoses, a fim de diminuir a contaminação ambiental visando minimizar os riscos de infecção por humanos (Robertson et al., 2000, Dall'agnol et al., 2010, Quadros et al., 2014). É importante este controle parasitológico e tratamento adequado e na suspeita de verminose, o clínico veterinário se acerca de exame para a tomada de decisão. O presente estudo teve por objetivo avaliar o parasitismo gastrointestinal em felinos atendidos no Hospital de Clínicas Veterinárias da UFRGS (HCV/UFRGS), no período de 2014 a 2016.

\section{Material e Métodos}

Amostras fecais de 339 felinos foram analisadas no período de 2014 ( $\mathrm{n}=163), 2015$ (n =97) e $2016(\mathrm{n}=79)$, provenientes do HCV/UFRGS. Dentre os animais atendidos, classificaram-se 307 adultos, 32 filhotes, 170 machos e 169 fêmeas. As amostras, recolhidas durante a consulta ou encaminhada posteriormente, foram acondicionadas em recipientes plásticos descartáveis de primeiro uso, identificadas e encaminhadas ao Laboratório de Helmintoses da FAVET/UFRGS, onde permaneceram conservadas a uma temperatura de $4^{\circ} \mathrm{C}$, sendo processadas num período máximo de 24 horas, pelos métodos de Willis-Mollay, de Lutz e de Faust (Hoffmann, 1987).

\section{Resultados e Discussão}

No período do estudo foram executados exames de fezes de 339 gatos. A positividade foi de $31,85 \%$ (108) com frequências de $35,6 \%$, $26,8 \%$ e $30,4 \%$, respectivamente, para 2014, 2015 e 2016. Os gêneros de parasitos diagnosticados nas fezes são mostrados na Tabela 1 e o resultado de gatos positivos, por gênero e faixa etária, é apresentado na Figura 1.

Em 85,2\% (93) dos felinos foi detectada a presença de animais com um gênero parasitário. Dentre os helmintos, destaca-se a predominância de Ancylostoma spp. (47,3\%), resultado semelhante ao encontrado em Goiânia (Campos et al., 1974), São Paulo (Côrtes et al., 1988, Silva et al., 2001), Santa Maria (Dall'agnol et al., 2010) e Londrina (Ferreira et al., 2013). 
Tabela 1. Resultado dos exames parasitológicos de felinos de Porto Alegre, RS, por ano e gênero parasitário

\begin{tabular}{|c|c|c|c|c|}
\hline Gêneros & $\begin{array}{c}2014 \\
\mathrm{n}\end{array}$ & $\begin{array}{c}2015 \\
\mathrm{n}\end{array}$ & $\begin{array}{c}2016 \\
n\end{array}$ & Total (\%) \\
\hline Ancylostoma & 34 & 7 & 3 & $44(40,74)$ \\
\hline Cystoisospora & 12 & 7 & 4 & $23(21,29)$ \\
\hline Toxocara & 2 & 6 & 6 & $14(12,96)$ \\
\hline Giardia & 2 & 1 & 3 & $6(5,55)$ \\
\hline Dipylidium & - & - & 3 & $3(2,77)$ \\
\hline Paragonimus & - & 1 & - & $1(0,92)$ \\
\hline Spirometra & - & - & 1 & $1(0,92)$ \\
\hline Trichuris & 1 & - & - & $1(0,92)$ \\
\hline Cystoisospora + Ancylostoma & 3 & - & - & $3(2,77)$ \\
\hline Ancylostoma + Trichuris & 3 & - & - & $3(2,77)$ \\
\hline Ancylostoma + Dipylidium . & - & 2 & - & $2(1,85)$ \\
\hline Cystoisospora + Toxocara & - & - & 2 & $2(1,85)$ \\
\hline Ancylostoma + Toxocara & - & 1 & - & $1(0,92)$ \\
\hline Dipylidium + Toxocara & - & - & 1 & $1(0,92)$ \\
\hline Dipylidium + Trichuris & - & 1 & - & $1(0,92)$ \\
\hline Giardia + Toxocara & 1 & - & - & $1(0,92)$ \\
\hline Cystoisospora + Ancylostoma + Toxocara & - & - & 1 & $1(0,92)$ \\
\hline Total & 58 & 26 & 24 & $108(100)$ \\
\hline
\end{tabular}

Em 85,2\% (93) dos felinos foi detectada a presença de animais com um gênero parasitário. Dentre os helmintos, destaca-se a predominância de Ancylostoma spp. (47,3\%), resultado semelhante ao encontrado em Goiânia (Campos et al., 1974), São Paulo (Côrtes et al., 1988, Silva et al., 2001), Santa Maria (Dall'agnol et al., 2010) e Londrina (Ferreira et al., 2013). Este parasita fixase a mucosa intestinal, através de seu aparelho bucal, causando ulceração da mucosa, acarretando um quadro de diarreia muco-sanguinolenta e anemia hemorrágica em felinos domésticos e silvestres; e dessa maneira, decorrendo emagrecimento, desidratação, depressão e diminuição nas atividades dos animais (Leite, 2012); é capaz de provocar em seres humanos uma doença denominada Larva Migrans Cutânea (LMC), sendo registrada principalmente em países de clima tropical e subtropical, e que está intimamente relacionada ao contato de adultos e crianças com areia de praças contaminadas, cujas larvas penetram através da pele, provocando erupções, na maioria das vezes de caráter pruriginoso (Santarém et al., 2004, Leite, 2012). Resultados divergentes foram demonstrados em São Paulo por Gennari et al. (2001), Ragozo et al. (2002), Funada et al. (2007) e por Stalliviere et al. (2009) Stalliviere et al. (2009).
As variações nos resultados devem-se, em parte, pela aplicação de diferentes técnicas laboratoriais no diagnóstico de cada gênero de parasito, e pela influência que o ambiente e as características de cada região exercem sobre a epidemiologia destes agentes parasitários ( $\underline{\text { Coelho }}$ et al., 2009). Entretanto, estes dados tornam-se importantes mesmo em baixa prevalência, pois helmintos do gênero Toxocara são capazes de levar animais jovens a óbito em consequência da acentuada infecção e migração excessiva de larvas, que provocam lesões hepáticas e pneumonia (Leite, 2012). Tais parasitos são considerados agentes zoonóticos, podendo causar no ser humano uma afecção denominada Larva Migrans Visceral (LMV), e que pode causar doenças mais graves devido a migração errática de algumas larvas, como encefalites e meningites. Um caso reportado na Itália por Caldera et al. (2013) comprovaram a infecção por Toxocara canis em um homem de 54 anos após a ingestão de caracóis crus infectados com os parasitos. Sinais como confusão mental, ataxia de membro periféricos, nistagmo, retenção urinária e mialgias foram observadas no paciente. $\mathrm{O}$ diagnóstico da infecção cerebral por T. canis veio por meio de sorologia positiva e detecção de DNA do parasito no líquido cefalorraquidiano. 


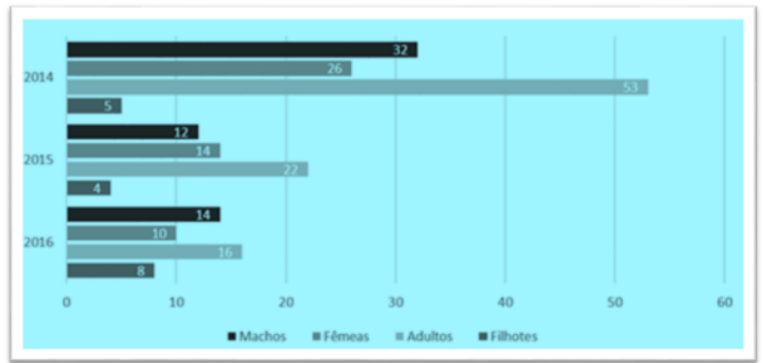

Figura 1. Resultado de amostras fecais positivas de felinos, por gênero, faixa etária e ano, no HCV/ UFRGS.

Cápsulas ovígeras de Dipylidium caninum foram diagnosticadas em 3,2\% dos gatos; prevalência semelhante $(3,1 \%)$ foi encontrada por Farias et al. (1995). Entretanto, Silva et al. (2001) registraram prevalência de $54,5 \%$, ambos os estudos no estado de São Paulo, cuja discrepância pode ser explicada pelo fato do diagnóstico realizado por Silva et al. (2001) ser obtido por necropsia, que evidencia as infecções por Dipylidium mais facilmente se comparadas a diagnósticos por exames de fezes (Gennari et al., 1999), que é também o caso do presente estudo. Elevadas infecções por Dipylidium podem acarretar irritação da mucosa intestinal, enterite, e prurido na região perianal devido a saída ativa de proglotes através do ânus (Leite, 2012).

A frequência de $24,73 \%$ de positividade para oocistos de Cystoisospora spp. foi semelhante a registrada por Ragozo et al. (2002), em amostras de fezes de gatos errantes de São Paulo $(26,1 \%)$ e Guarulhos (24,6\%). Dados similares foram apontados também por Gennari et al. (1999), em gatos domiciliados de diferentes áreas de São Paulo, com positividade de $38,5 \%$. A contaminação do meio ambiente somada a falta de saneamento básico tornam-se importantes fatores contribuintes para a ocorrência desta afecção, sendo seu controle dificultado devido à facilidade na disseminação do agente etiológico (Leite, 2012). A associação mais frequente foi de Cystoisospora spp. e Ancylostoma spp. (2,77\%) resultado semelhante foi apresentado por Coelho et al. (2009) para felinos no município de Andradina, São Paulo.

Cistos de Giardia foram encontrados em $6,45 \%$ das amostras fecais analisadas, com resultado semelhante $(5,9 \%)$ relatado por Coelho et al. (2009). Giardia é frequentemente referido como um protozoário entérico comum entre cães e gatos, entretanto sua prevalência é geralmente subestimada devido à baixa sensibilidade de métodos de diagnósticos empregados na rotina laboratorial (McGlade et al., 2003).
Habitualmente tem sido relatado como responsável por causar quadros de diarreia em gatos, especialmente em filhotes; contudo, seu verdadeiro potencial ainda não foi esclarecido (Leite, 2012); a ocorrência de sintomas é manifestada geralmente em animais jovens e/ou mantidos em ambientes estressantes (Quadros et al., 2015)), podendo variar de diarreia e perda de peso à desidratação e anorexia (Cavalini and Zappa, 2011). Sendo este parasito um agente zoonótico, torna-se de grande importância seu diagnóstico e controle, a fim de reduzir as fontes de infecção.

No presente estudo foram encontrados ovos de Paragonimus spp. em um felino $(1,1 \%)$ no ano de 2015, e chamou a atenção pela característica zoonótica do agente, sendo discutido com o veterinário na clínica, cujas informações foram compartilhadas com o proprietário do gato. Gatos é fonte de infecção contaminando o meio ambiente com ovos liberados com as fezes (Figura 2).

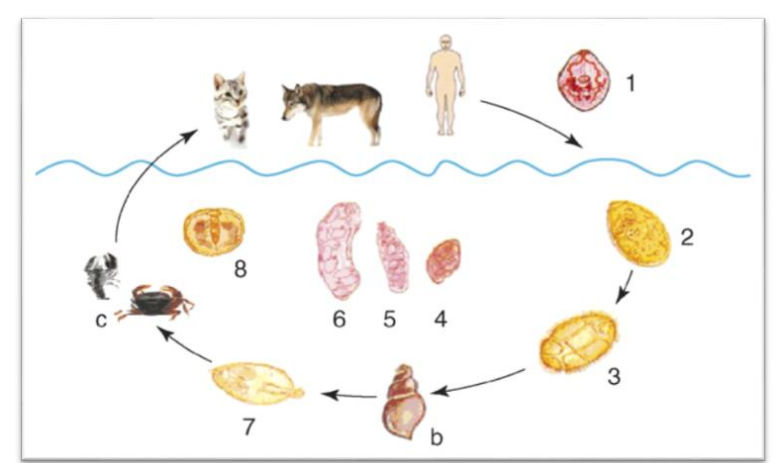

Figura 2. Ciclo biológico de Paragonimus westermani. No hospedeiro definitivo, parasito adulto (1) nos pulmões. Ovo (2) liberado via esputo e fezes. Miracidia na água (3) penetra no caramujo (b), o primeiro hospedeiro intermediário. Miracidia dentro do caramujo passa por diversos estágios de desenvolvimento [esporocisto $(4)$, rédia $(5,6)$ ] e cercária desenvolvida (7), que vai para a água em busca do segundo hospedeiro - caranguejos e lagostas - (c), no qual a cercária se torna a metacercáriaincistada (8) na musculatura e outros órgãos, cuja ingestão resulta na infecção no hospedeiro definitivo. Fonte: Liu et al. (2008).

A paragonimíase é considerada uma doença cosmopolita que apresenta evolução crônica acometendo principalmente o pulmão. É uma parasitose pouco conhecida; entretanto é considerada uma zoonose alimentar na China (Liu et al., 2008) e sua principal via de infecção é através da ingestão de crustáceos de água doce crus, malcozidos ou em conserva (Siqueira-Batista et al., 2006). Seu primeiro registro ocorreu no Brasil em lontra (Lutra braziliense) em 1950. Carnívoros são hospedeiros reservatórios do 
parasito, sendo definitivos com humanos, cães e suínos e uma variedade de felídeos. O diagnóstico definitivo é baseado na identificação de ovos nas fezes e esputo. A alta prevalência desta parasitose na China pode refletir, em parte, ao inadequado controle e estratégias para sua prevenção (Ruan, 2006).

Parasitos do gênero Spirometra são raramente identificados em exames coprológicos no sul do Brasil (Dall'agnol et al., 2010). Ele é o causador da infecção denominada esparganose, cujas larvas plerocercóides são responsáveis pela aparição de nódulos subcutâneos nos olhos, cérebro e medula espinhal, sendo humanos seus hospedeiros acidentais, enquanto gatos e outros animais são hospedeiros definitivos (Chung et al., 2012). Em estudo realizado no centro de controle de zoonoses do município de Uberlândia, Mundim et al. (2004) verificaram uma prevalência de $4 \%$ para Spirometra mansonoides, resultado similar foi encontrado por (Dall'agnol et al., 2010), onde 116 amostras fecais de gatos domiciliados da cidade de Santa Maria, no estado do Rio Grande do Sul, foram analisadas, verificando-se uma porcentagem de $2,6 \%$ destes parasitos, ambos os resultados se assemelham aos encontrados no presente estudo, no qual $1,1 \%$ dos animais apresentavam-se infectados. Os gêneros Paragonimus e Spirometra demandam atenção devido seu potencial zoonótico, o que torna o papel do médico veterinário de extrema importância para diagnóstico e tratamento destas parasitoses, evitando a transmissão e acometimento de doenças sérias por seres humanos, bem como a difusão e conscientização sobre as parasitoses zoonóticas.

\section{Referências Bibliográficas}

Caldera, F., Burlone, M. E., Genchi, C., Pirisi, M. \& Bartoli, E. 2013. Toxocara encephalitis presenting with autonomous nervous system involvement. Infection, 41, 691-694.

Campos, D. B., Garibaldi, I. M. \& Carneiro, J. R. 1974. Prevalência de helmintos em gatos (Felis catus domesticus) de Goiânia. Revista de Patologia Tropical, 3, 355-359.

Cavalini, P. P. \& Zappa, V. 2011. Giardíase felina - revisão de literatura. Revista Científica Eletrônica de Medicina Veterinária, IX, 1-18.

Chung, S. W., Kim, Y. H., Lee, E. J., Kim, D. H. \& Kim, G. Y. 2012. Two cases of pulmonary and pleural sparganosis confirmed by tissue biopsy and immunoserology. Brazilian Journal of Infectious Diseases, 16, 200-203.

Coelho, W. M. D., Amarante, A. F. T. d., Soutello, G., Velludo, R., Meireles, M. V. \& Saraiva Bresciani, K. D. 2009. Ocorrência de parasitos gastrintestinais em amostras fecais de felinos no município de Andradina, São Paulo. Revista Brasileira de Parasitologia Veterinária, 18, 46-49.

Côrtes, V. d. A., Paim, G. V. \& Alencar Filho, R. A. 1988. Infestação por ancilostomídeos e toxocarídeos em cães e gatos apreendidos em vias públicas, São Paulo (Brasil). Revista de Saúde Pública, 22, 341-343.

Dall'agnol, L. P., Otto, M. A., Silva, A. S. \& Monteiro, S. G. 2010. Parasitos gastrintestinais em gatos naturalmente infectados no município de Santa Maria no estado do Rio Grande do Sul, Brasil. Acta Veterinaria Brasilica, 4, 181184.

Farias, N. A., Christóvão, M. L. \& Stobbe, N. S. 1995. Frequiência de parasitas intestinais em cães (Canis familiaris) e gatos (felis catus domestica) em Araçatuba, São Paulo. Revista Brasileira de Parasitologia Veterinária, 4, 5760.

Ferreira, F. P., Dias, R. C. F., Martins, T. A., Constantino, C., Pasquali, A. K. S., Vidotto, O., Freire, R. L. \& Navarro, I. T. 2013. Frequência de parasitas gastrointestinais em cães e gatos do município de Londrina, PR, com enfoque em saúde pública. Semina: Ciências Agrárias, 34, 3851-3858.

Funada, M. R., Pena, H. F. J., Soares, R. M., Amaku, M. \& Gennan, S. M. G. 2007. Freqüência de parasitos gastrintestinais em cães e gatos atendidos em hospital-escola veterinário da cidade de São Paulo. Arquivo Brasileiro de Medicina Veterinária $e$ Zootecnia, 59, 1338-1340.

Gennari, S. M., Kasai, N., Pena, H. F. d. J. \& Cortez, A. 1999. Occurrence of protozoa and helminths in faecal samples of dogs and cats from São Paulo city. Brazilian Journal of Veterinary Research and Animal Science, 36, $1-4$.

Gennari, S. M., Pena, H. F. J. \& Blasques, L. S. 2001. Freqüência de ocorrência de parasitos gastrintestinais em amostras de fezes de cães e gatos da cidade de São Paulo. Veterinária News, 8, 10-12.

Hoffmann, R. P. 1987. Diagnóstico de parasitismo veterinário. Sulina, Porto Alegre, Brasil. 
Leite, L. C. 2012. Ocorrência de endoparasitas com potencial zoonótico de transmissão em fezes de gatos (Felis catus domesticus Linnaeus, 1758) domiciliados na área urbana e região metropolitana de Castro-Paraná-Brasil. Ambiência, 8, 923-930.

Liu, Q., Wei, F., Liu, W., Yang, S. \& Zhang, X. 2008. Paragonimiasis: an important food-borne zoonosis in China. Trends in Parasitology, 24, 318-323.

McGlade, T. R., Robertson, I. D., Elliot, A. D., Read, C. \& Thompson, R. C. A. 2003. Gastrointestinal parasites of domestic cats in Perth, Western Australia. Veterinary Parasitology, 117, 251-262.

Mundim, T. C. D., Oliveira Júnior, S. D., Rodrigues, D. C. \& Cury, M. C. 2004. Frequency of helminthes parasites in cats of Uberlândia, Minas Gerais. Arquivo Brasileiro de Medicina Veterinária e Zootecnia, 56, 562563.

Quadros, R. M., Liz, F. R. \& Marques, S. M. T. 2014. Ocorrência de ovos de Toxocara spp. em solos de praças públicas de Lages, Santa Catarina. Ars Veterinaria, 30, 109-114.

Quadros, R. M., Weiss, P. H. E., Miletti, L. C., Ezequiel, G. W. \& Marques, S. M. T. 2015. Ocorrência de Giardia duodenalis em cães domiciliados e apreendidos pelo Centro de Controle de Zoonoses de Lages, Santa Catarina, Brasil. Revista Portuguesa de Ciências Veterinárias, 110, 127-132.

Ragozo, A. M. A., Muradian, V., Ramos e Silva, J. C., Caravieri, R., Amajoner, V. R., Magnabosco, C. \& Gennari, S. M. 2002. Ocorrência de parasitos gastrintestinais em fezes de gatos das cidades de São Paulo e Guarulhos. Brazilian Journal of Veterinary Research and Animal Science, 39, 244-246.

Robertson, I. D., Irwin, P. J., Lymbery, A. J. \& Thompson, R. C. A. 2000. The role of companion animals in the emergence of parasitic zoonoses. International Journal for Parasitology, 30, 1369-1377.
Ruan, W. 2006. Epidemiology survey of paragonimiasis in several counties of Zhejiang Province during 2000-2005. Practical Preventive Medicine, 13, 1488-14489.

Santarém, V. A., Giuffrida, R. \& Zanin, G. A. 2004. Larva migrans cutânea: ocorrência de casos humanos e identificação de larvas de Ancylostoma spp em parque público do município de Taciba, São Paulo. Revista Brasileira de Medicina Tropical, 37, 179-181.

Schantz, P. M. 1991. Parasitic zoonoses in perspective. International Journal for Parasitology, 21, 161-170.

Silva, H. C., Castagnolli, K. C., Silveira, D. M., Costa, G. H. N., Gomes, R. A. \& Nascimento, A. A. 2001. Fauna helmíntica de cães e gatos provenientes de alguns municípios do Estado de São Paulo. Semina: Ciências Agrárias, 22, 67-71.

Siqueira-Batista, R., Gomes, A. P., Albuqueque, V. S., de-Pina-Costa, A., Rocha-Mello, A., Guimarães-Pereira, E., Ramos-Oliveira, F. \& Rubião, E. C. N. 2006. História natural da infecção por Paragonimus: abordagem clínica e ecológica. Revista Pulmão RJ, 15, 270-276.

Stalliviere, F. M., Bellato, V., Souza, A. P., Sartor, A. A., Moura, A. B. \& Dalla Rosa, L. 2009. Ectoparasitos e helmintos intestinais em Felis catus domesticus, da cidade de Lages, SC, Brasil e aspectos sócioeconômicos e culturais das famílias dos proprietários dos animais. Revista Brasileira de Parasitologia Veterinária, 18, 26-31.

Tesserolli, G. L., Fayzano, L. \& Agottani, J. V. B. 2005. Ocorrência de parasitas gastrintestinais em fezes de cães e gatos, Curitiba-PR. Revista Acadêmica, 3, 31-34.

Article History:

Received 17 July 2017

Accepted 16 August 2017

Available on line 8 September 2017

License information: This is an open-access article distributed under the terms of the Creative Commons Attribution License 4.0, which permits unrestricted use, distribution, and reproduction in any medium, provided the original work is properly cited. 